\title{
Author Correction: An ultrahot Neptune in the Neptune desert
}

James S. Jenkins (D, Matías R. Díaz, Nicolás T. Kurtovic, Néstor Espinoza Đ, Jose I. Vines, Pablo A. Peña Rojas, Rafael Brahm (D), Pascal Torres, Pía Cortés-Zuleta (D), Maritza G. Soto, Eric D. Lopez, George W. King, Peter J. Wheatley (D), Joshua N. Winn (1), David R. Ciardi, George Ricker, Roland Vanderspek, David W. Latham, Sara Seager (D), Jon M. Jenkins (1), Charles A. Beichman, Allyson Bieryla (1), Christopher J. Burke, Jessie L. Christiansen (D, Christopher E. Henze,

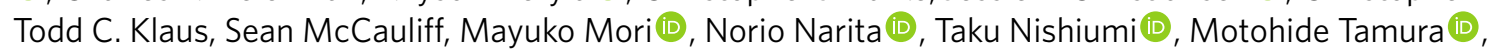
Jerome Pitogo de Leon, Samuel N. Quinn, Jesus Noel Villaseñor, Michael Vezie, Jack J. Lissauer (1), Karen A. Collins (D), Kevin I. Collins (D), Giovanni Isopi, Franco Mallia, Andrea Ercolino, Cristobal Petrovich, Andrés Jordán, Jack S. Acton, David J. Armstrong (D), Daniel Bayliss (10), François Bouchy, Claudia Belardi, Edward M. Bryant, Matthew R. Burleigh,

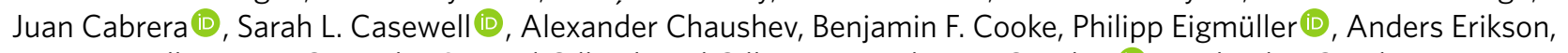
Emma Foxell, Boris T. Gänsicke, Samuel Gill, Edward Gillen, Maximilian N. Günther (D), Michael R. Goad, Matthew J. Hooton (10, James A. G. Jackman, Tom Louden, James McCormac, Maximiliano Moyano, Louise D. Nielsen,

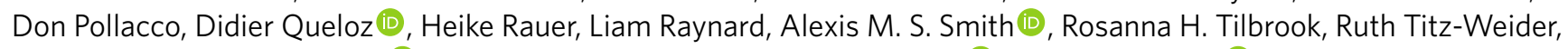

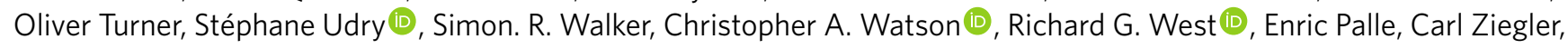
Nicholas Law and Andrew W. Mann

Correction to: Nature Astronomy https://doi.org/10.1038/s41550-020-1142-Z, published online 21 September 2020.

In the version of this Letter originally published, the present address of Rafael Brahm, 'Facultad de Ingeniería y Ciencias, Universidad Adolfo Ibáñez, Peñalolén, Chile', was not listed. This has now been corrected.

Published online: 13 October 2020

https://doi.org/10.1038/s41550-020-01252-5

๑) The Author(s), under exclusive licence to Springer Nature Limited 2020 